\title{
PNPRS OFFICERS AND MEMBERS, 2019
}

\section{Officers}

1. Dr. Jove Jim S. Aguas (University of Santo Tomas, Manila) - Executive Governor

2. Dr. Juan Rafael G. Macaranas (De La Salle-College of Saint Benilde, Manila) - Chairman

3. Dr. Danilo S. Alterado (Saint Louis University, Baguio) - Vice Chairman

4. Dr. Jeffrey L. Bartilet (Polytechnic University of the Philippines, Manila) - Secretary

5. Mr. Alvin O. Tan (University of Santo Tomas, Manila) - Treasurer

6. Dr. Fleurdeliz R. Altez-Albela (University of Santo Tomas, Manila) - Auditor

7. Dr. Rodrigo D. Abenes (Philippine Normal University, Manila) - Public Relations Officer

8. Ms. Ma. Glovedi Joy L. Bigornia (Philippine Military Academy, Baguio) - Legal Officer

9. Ms. Basilisa P. Macaranas-Administrative Officer

\section{Philosophia Editorial Board Members}

1. Dr. Edward Alam (Notre Dame University, Louaize, Lebanon)

2. Dr. Paolo A. Bolaños (University of Santo Tomas)

3. Dr. Peter M. Collins (Maryland, USA)

4. Dr. Noelle Leslie Dela Cruz (De La Salle University, Manila)

5. Dr. Yousuf Dadoo (University of South Africa, Pretoria)

6. Dr. Tran van Doan (National Taiwan University, Taipei)

7. Dr. Leni dlR. Garcia (De La Salle University, Manila)

8. Dr. Lok Chong Hoe (University Sains Malaysia, Penang)

9. Dr. Ted Honderich (University College London, UK)

10. Dr. Daniel P. Kroger (St. Anthony Messenger Press, Ohio, USA)

11. Dr. James M. Lawler (Retired, State University of New York, Buffalo, USA)

12. Dr. Napoleon M. Mabaquiao, Jr. (De La Salle University, Manila)

13. Dr. Halina Marlewicz (Jagiellonian University, Kraków, Poland)

14. Dr. Zailan Moris (Universiti Sains Malaysia, Penang)

15. Dr. George Mousourakis (Ritsumeikan University, Kyoto)

16. Dr. Olatunji Oyeshile (University of Ibadan, Nigeria)

17. Dr. Narcisa Paredes-Canilao (University of the Philippines, Baguio City)

18. Dr. Santiago Sia (Retired, Milltown Institute, National University of Ireland, Dublin)

19. Dr. Warayuth Sriwarakuel (Assumption University of Thailand, Bangkok)

20. Dr. William Sweet (St. Francis Xavier University, Nova Scotia, Canada)

21. Dr. Brendan Sweetman (Rockhurst University, Kansas, Missouri, USA)

22. Dr. Paul Thom (University of Sydney, Australia)

23. Dr. Wilfried Ma. Vanhoutte (Saint Louis University, Baguio City) 
24. Dr. Mathew Varghese (The Eastern Institute \& Aoyama Gakuin University, Tokyo, Japan)

25. Dr. Jeanette Yasol-Naval (University of the Philippines, Diliman, Quezon City)

\section{Members}

1. Ms. Aireen T. Abiera (Marikina Polytechnic College, Marikina City)

2. Dr. Carmelito Nomer S. Abolencia (Eastern Visayas State University, Tacloban City)

3. Dr. Victor R. Aguilan (Silliman University, Dumaguete)

4. Ms. Ninotchka Mumtaj B. Albano (De La Salle University, Manila)

5. Dr. Albert C. Albina (Negros Oriental State University, Bayawan-Sta. Catalina)

6. Dr. Wilhelm Julian Alfredo (Department of Agrarian Reform, Quezon City)

7. Dr. Marián Ambrozy (College of International Business ISM, Slovakia)

8. Dr. Gabriel Andrade (St. Matthew's University School of Medicine, Cayman Islands)

9. Dr. Emmanuel Ifeanyi Ani (University of Ghana, Legon)

10. Mr. Julius O. Basallo (Cebu Technological University-Barili Campus)

11. Mr. Dennis M. Bautista (Leyte Normal University, Tacloban City)

12. Mr. Henry P. Bilan (Kalinga State University, Kalinga)

13. Ms. Roxanne T. Bongco (Bataan Peninsula State University)

14. Mr. Elmer Palencia Brabante (Polytechnic University of the Philippines, Manila)

15. Dr. Mark Joseph Calano (Ateneo de Manila University, Quezon City)

16. Prof. Raşit Çelik (Department of Educational Sciences, Trabzon University, Turkey)

17. Mr. Delfin P. Chiniona (Technological Institute of the Philippines, Manila)

18. Dr. Dalmacito A. Cordero, Jr. (De La Salle University, Manila)

19. Dr. Franz Giuseppe F. Cortez (University of Santo Tomas, Manila)

20. Mr. Jose Fermin B. Crave (Cebu Normal University, Cebu City)

21. Dr. Robelyn P. Cunanan (Batangas State University-Main I, Batangas City)

22. Dr. Charlie M. Dagwasi (Benguet State University)

23. Mr. Zacarias G. Damo, Jr. (Mariano Marcos State University)

24. Ms. Patricia Amanda J. Dela Cruz (University of the Philippines-Diliman)

25. Dr. Noelle Leslie G. Dela Cruz (De La Salle University, Manila)

26. Fr. Prudencio M. Edralin, Ph.D. (Thomas Aquinas Major Seminary/Lyceum of Aparri)

27. Mr. Beljun P. Enaya (Visayas State University, Baybay City)

28. Fr. Francis Paul Escaño, RCJ (Rogationist Seminary College, Cebu City)

29. Mr. Joven B. Esteban (Holy Angel University, Angeles City, Pampanga)

30. Dr. Edwin Etieyibo (University of Witwatersrand, Johannesburg, South Africa)

31. Mr. Roy John R. Del Rosario (Centro Escolar University, Manila)

32. Dr. Guiraldo C. Fernandez, Jr. (Visayas State University, Baybay City)

33. Mr. Aldrin Mathew L. Go

34. Mr. Aris S. Gonzales (Holy Angel University, Angeles City, Pampanga)

35. Dr. Eden M. Gripaldo (Retired) (University of the Philippines-Diliman, Quezon City)

36. Ms. Veniz Maja V. Guzman (De La Salle University, Manila) 
37. Ms. Ma. Rubeth Hipolito (Holy Angel University, Angeles City, Pampanga)

38. Mr. Aldrin S. Jaramilla (University of Northern Philippines, Vigan City)

39. Mr. Joseph Martin M. Jose (De La Salle University, Manila)

40. Dr. Danilo L. Libradilla (Cebu Technological University-Barili Campus)

41. Dr. Leslie Anne L. Liwanag (Visayas State University, Baybay City)

42. Dr. Miloš Lokajíček (Institute of Physics of the Czech Academy of Sciences)

43. Mr. Edward L. Macariola (Saint Louis University, Baguio City)

44. Mr. Welfredo Q. Mamaril (Holy Angel University, Angeles City, Pampanga)

45. Mr. Edmund M. Maniago (Holy Angel University, Angeles City, Pampanga)

46. Mr. Angelito L. Martinez (Holy Angel University, Angeles City, Pampanga)

47. Dr. Rhochie Avelino E. Matienzo (University of Santo Tomas, Manila)

48. Dr. Robert A. Montaña (University of Santo Tomas, Manila)

49. Mr. Romeo F. Narvaez (Mindanao State University, General Santos City)

50. Dr. Ngoc Thi Minh Nguyen (Institute of Sociology, Ha Noi, Vietnam)

51. Mr. Marc Benigno C. Olaguir (Bohol State University, Bohol)

52. Dr. Ian Raymond B. Pacquing (University of Santo Tomas, Manila)

53. Mr. Joseph P. Paña (De La Salle University, Manila)

54. Dr. Engelbert C. Pasag (PanPacific University, Urdaneta City)

55. Mr. John Paul J. Petrola (University of San Agustin, Iloilo City)

56. Dr. Joezenon A. Purog (Eastern Visayas State University, Tacloban City)

57. Mr. Virgilio A.Rivas (Polytechnic University of the Philippines, Manila)

58. Mr. Beaujorne Sirad A. Ramirez (Immaculate Conception Academy, Greenhills)

59. Dr. Christine Carmela R. Ramos (Mapua University, Manila)

60. Dr. Beverly A. Sarza (De La Salle University)

61. Dr. Alfredo B. Santos (Holy Angel University, Angeles City, Pampanga)

62. Mr. Mark Louie Tabunan (University of Northern Philippines, Ilocos Sur)

63. Dr. Alex Y. Tiempo (Cebu Normal University, Cebu City)

64. Mr. Jose Z. Tria (Catanduanes State University)

65. Dr. Michal Valčo (University of Presov in Presov, Slovakia)

66. Mr. Benito T. Villareal III (Jose Rizal College)

67. Mr. Al Franjon M. Villaroya (Visayas State University, Baybay City) 